\title{
Cytoplasmic influence on the expression of nuclear genes affecting life span in Drosophila melanogaster
}

\author{
ISAMU YONEMURA, TOMIO MOTOYAMA, * HAYATO HASEKURA \& \\ BARRY BOETTCHER $\dagger$ \\ Department of Legal Medicine, Shinshu University School of Medicine, Matsumoto, 390 Japan, *Department of \\ Physiology, College of Environmental Health, Azabu University, Sagami-hara, 229 Japan and †Department of Biological \\ Sciences, The University of Newcastle, New South Wales, 2308 Australia
}

\begin{abstract}
In earlier studies we have found that the difference between short and long life spans of two inbred strains of Drosophila melanogaster is controlled by nuclear major genes. The present study has revealed a cytoplasmic factor that influences the expression of the nuclear longevity genes. The factor shows a typical maternal inheritance and is considered to be an extranuclear gene, such as mitochondrial DNA (chondriome). This paper marks the discovery of two basic forms of inheritance, nuclear and extra-nuclear, in the genetics of life span of $D$. melanogaster. These findings suggest that further studies, including genetic engineering, on longevity and aging might enable direct manipulation of these characters.
\end{abstract}

Keywords: Drosophila melanogaster, extranuclear genes, gene expression, life-span, nuclear major genes.

\section{Introduction}

From the results obtained in a number of studies, it has been concluded that life span is under polygenic control. Genetic contribution to variation of life span is not large; environmental conditions such as temperature or food are major determinants. Heritability of life span has been calculated as 0.133-0.442 in Drosophila (Maynard Smith, 1959; Tantawy \& Rakha, 1964), 0.2-0.3 in nematodes (Johnson \& Wood, 1982), and $0.21-0.36$ in mice (Storer, 1966).

Goodrick (1975), however, obtained high heritability (0.47-0.787) of life span in mice, and his calculations indicated that the inheritance of life span in mice was controlled by a single gene. The present authors have undertaken mating experiments with two purebred strains of Drosophila melanogaster (D.m.) and have demonstrated that major genes at two loci, autosomal and X chromosomal, determined their life spans. These longevity genes were analysed for their mode of inheritance and were named Jm (Yonemura et al., 1989, 1990). Luckinbill et al. (1988) also succeeded in selection of life span in D.m., and demonstrated that the difference in life span in their stocks was mainly determined by a few genes on chromosome 1 (X chromosome) and chromosome 3 (autosome).
The present work reports the discovery of a new genetic influence on life span of D.m. involving extranuclear genes as well as nuclear genes, Jm A and Jm X. Thus, two fundamental modes of inheritance, nuclear and extranuclear, are shown to influence life span.

\section{Materials and methods}

Two short-lived purebred strains of D.m. were used as the parental stocks, $P_{1}$ and $P_{2}$. These were mated according to the combinations listed in Table 1 to produce reciprocal $F_{1}$ hybrids, $F_{2}$ and all backcross generations. Flies were bred at $27^{\circ} \mathrm{C}$ on food containing 8 per cent dry yeast and 5 per cent sugar. The dead were counted every day and were removed from the culture vials. The $P_{1}$ strain was established from a wild type stock of D.m. through 13 or more generations of sib-mating; their genes are presumed to be homozygous at 95 per cent or more loci. This strain is identical with the $\mathrm{P}_{1}$ generation (the short-lived parent) used in previous work (Yonemura et al., 1989, 1990). The $\mathrm{P}_{2}$ strain in the present work is a purebred line established by the same procedure from Oregon R. Details of culture and handling methods were the same as in an earlier report (Yonemura et al., 1989). 


\section{Results}

The means and standard deviations of the parental stocks, the $F_{1}$ and $F_{2}$ generations, and the reciprocal backcross generations are given in Table 2. These data ignore the obvious differences between the sexes or between different crosses in the same generations. However, the procedure is necessary to enable the following calculations.

If we take the phenotypic variance in the $\mathrm{F}_{2}$ as $V_{\mathrm{P}}$, the genotypic variance as $V_{\mathrm{G}}$, and the environmental variance as $V_{\mathrm{E}}$, then $V_{\mathrm{P}}=V_{\mathrm{G}}+V_{\mathrm{E}} . V_{\mathrm{E}}$ is given by $\left(V_{\mathrm{P}_{1}}+V_{\mathrm{P}_{2}}+2 V_{\mathrm{F}_{1}}\right) / 4$ using variances of $\mathrm{P}_{1}, \mathrm{P}_{2}$ and $\mathrm{F}_{1}$. From Table 2 it can be calculated that $V_{\mathrm{P}}=289.00$ and $V_{\mathrm{E}}=153.05$. Heritability in the broad sense, $h^{2} b$, is given by $V_{\mathrm{G}} / V_{\mathrm{P}}$. Then $h^{2} b$ can be calculated as $h^{2} b=0.4704$. $V_{\mathrm{G}}$ is divided into additive variance $\left(V_{\mathrm{A}}\right)$, dominance variance $\left(V_{\mathrm{D}}\right)$ and interaction variance $\left(V_{\mathrm{I}}\right): V_{\mathrm{G}}=V_{\mathrm{A}}+V_{\mathrm{D}}+V_{\mathrm{I}} . V_{\mathrm{A}}$ is given by $2 V_{\mathrm{F}_{2}}-V_{\mathrm{B}}$, which is calculated from Table 2 as $V_{\mathrm{A}}=305.75$. This figure is larger than $V_{\mathrm{G}}=135.95$ calculated before, indicating that $V_{\mathrm{D}}$ and $V_{\mathrm{I}}$ take no part in $V_{\mathrm{G}}$. In such a genetic model the number of loci of the contributing genes can be calculated as $N=R^{2} / 8 V_{\mathrm{A}}=0.03$ (where $R$ is the difference of mean life span between $P_{1}$ and $\left.P_{2}\right)$.

Table 1 Notation of generations in reciprocal crosses

\begin{tabular}{|c|c|}
\hline Mating & Progeny \\
\hline$P_{1} \sigma \times P_{2}$ ᄋ & $F_{1-1}$ \\
\hline$P_{1}$ O $\times P_{2} \sigma^{\prime \prime}$ & $F_{1-2}$ \\
\hline$F_{1-1} \sigma \times F_{1-1}$ ㅇ & $F_{2-1}$ \\
\hline$F_{1-2} ठ F_{1-2}$ ㅇ & $F_{2-2}$ \\
\hline$P_{1} \sigma \times F_{1-1}$ ㅇ & $B_{1-1}$ \\
\hline$P_{1} \sigma \times F_{1-2}$ ? & $B_{1-2}$ \\
\hline$P_{1}$ 우 $\times F_{1-1}$ ठ & $B_{1-3}$ \\
\hline$P_{1}$ O $\times F_{1-2} \sigma$ & $B_{1-4}$ \\
\hline$P_{2} \sigma \times F_{1-1}$ ㅇ & $B_{2-1}$ \\
\hline$P_{2} \sigma \times F_{1-2}$ 우 & $B_{2-2}$ \\
\hline$P_{2} \% \times F_{1-1} \sigma$ & $B_{2-3}$ \\
\hline$P_{2} Q \times F_{1-2} \sigma$ & $B_{2-4}$ \\
\hline
\end{tabular}

Table 2 Sample number in each generation and the mean life span. Values are means $\pm s . d$. (days)

\begin{tabular}{lcc}
\hline Generation & Number & Life span \\
\hline$P_{1}($ ठ + ○) & 641 & $36.5 \pm 5.6$ \\
$P_{2}($ ठ +@) & 640 & $28.1 \pm 3.1$ \\
$F_{1}$ (total) & 1297 & $48.1 \pm 16.9$ \\
$F_{2}$ (total) & 1303 & $43.4 \pm 17.0$ \\
$B_{1}$ (total) & 2539 & $47.0 \pm 15.8$ \\
$B_{2}($ total $)$ & 2582 & $39.8 \pm 16.3$ \\
$B$ (total) & 5121 & $43.4 \pm 16.5$ \\
\hline
\end{tabular}

These results show that the inheritance of life span in this experiment was controlled by few major genes.

On the other hand, the scaling test of Mather (1949) requires the following calculations, taking the means of $B_{1}, B_{2}$ and $F_{2}$ generations as $\overline{B_{1}}, \overline{B_{2}}$ and $\overline{F_{2}}$ and those of $P_{1}, P_{2}$ and $F_{1}$ as $\overline{P_{1}}, \overline{P_{2}}$ and $\overline{F_{1}}: A=2 \overline{B_{1}}-\overline{P_{1}}-\overline{F 1}$, $B=2 \overline{B_{2}}-\overline{P_{2}}-\overline{F_{1}}, C=4 \overline{F_{2}}-2 \overline{F_{1}}-\overline{P_{1}}-\overline{P_{2}}$.

If $A \fallingdotseq B \fallingdotseq C \fallingdotseq 0$, genes in the model are considered to have only additive and dominance effects and to show no epistatic effect; epistatic effect is an interaction between gene loci. Calculations based on the data in Table 2 result in $A=9.4 \pm 0.814, B=3.4 \pm 0.804$ and $C=12.8 \pm 2.12$, which are significantly larger than $0(P<0.05)$. This shows interaction variance is large. This is inconsistent with the above conclusion that $V_{\mathrm{G}}$ solely consists of $V_{\mathrm{A}}$. Consequently, the quantitative genetic analysis is invalid.

The distribution of life spans of the different generations of D.m. studied here are shown in Fig. $1\left(P, F_{1}\right.$ and $F_{2}$ generations) and Fig. 2 (backcross generations).

The progenies showing the shortest mean life spans were $P_{2}$ and generations originating from $P_{2}$ females: $F_{1-1}, F_{2-1}, B_{1-1}, B_{2-1}, B_{2-3}, B_{2-4}$. The exception to this general observation was $B_{1-3}$. It is pointed out that, although $P_{2}$ females in the parental generation initiated the $B_{1-3}$ generation, the females in the backcross $\left(P_{1}\right)$ contained no contribution (either nuclear or cytoplasmic) from $P_{2}$ females.

In the progenies where the females used for breeding did not have a contribution from $P_{2}$ females, $F_{1-2}$, $F_{2-2}, B_{1-2}, B_{1-3}, B_{1-4}, B_{2-2}$, long-lived flies were observed. Such long-lived flies were not observed among the parental strains, including $P_{1}$ which, similarly, did not have a contribution from $P_{2}$ females.

\section{Discussions and conclusions}

The striking observation in this study is the marked difference in the life spans of progenies from reciprocal crosses. For example, $F_{1-1}$ and $F_{1-2} ; F_{2-1}$ and $F_{2-2}$ (Fig. 1). A difference between the mean life spans of male $F_{1}$ flies from reciprocal crosses might be accounted for by differences in genes located on the $\mathrm{X}$ and $Y$ chromosomes of the parental stocks. However, such an explanation could not account for the difference in the mean life spans of female $F_{1}$ flies observed here $\left(F_{1-1}\right.$ and $F_{1-2}$, Fig. 1). However, an extranuclear influence appears to be involved. All progenies where the cytoplasm of the zygote originated from the $P_{2}$ parental strain, $P_{2}, F_{1-1}, F_{2-1}, B_{1-1}, B_{2-1}, B_{2-3}$, $B_{2-4}$, showed a unimodal, short, life span. On the other hand, progenies where the cytoplasm of the zygote originated from the $P_{1}$ parental strain, but the nuclear genetic material contained a contribution from the $P_{2}$ 


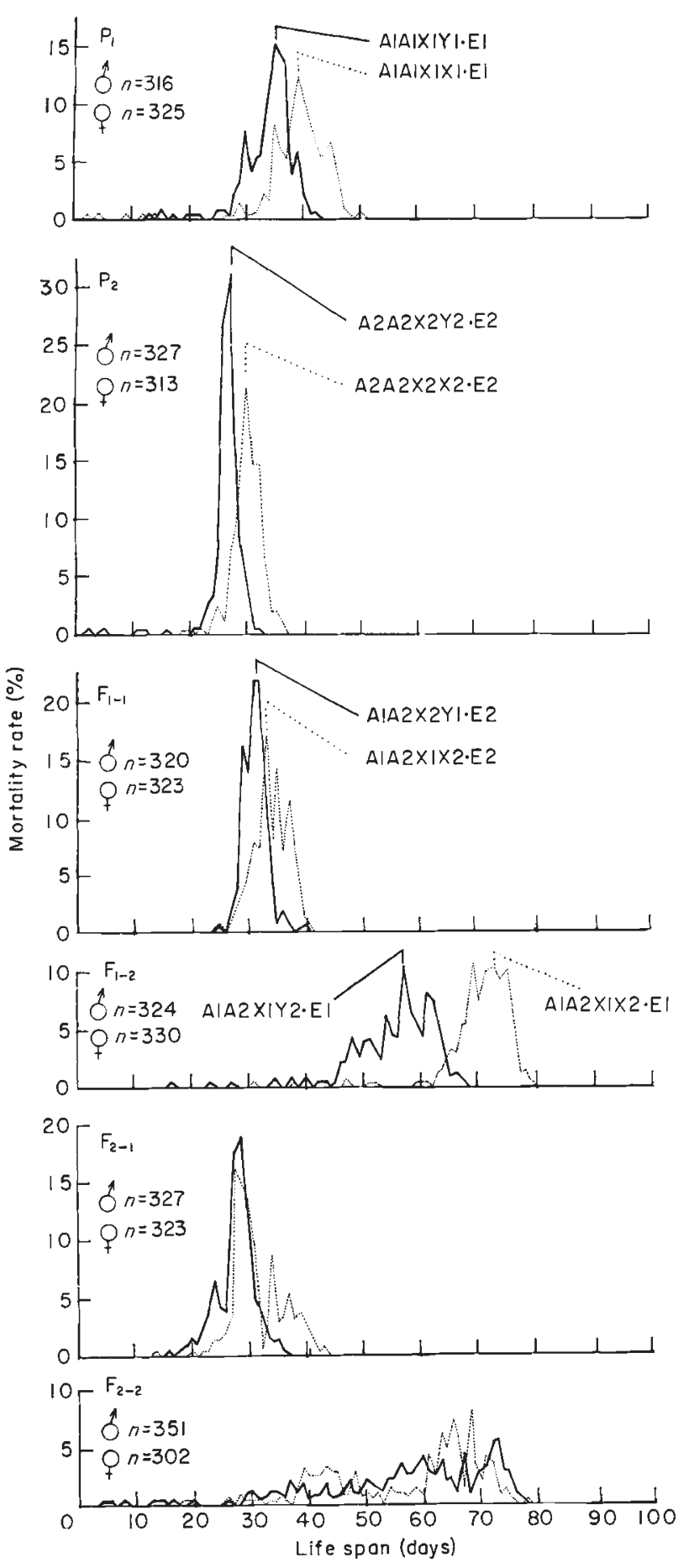

Fig. 1 Life spans of stocks of Drosophila melanogaster, where day of emergence is given as day 0 .

Distributions of mortality rates in parent generations $\left(P_{1}, P_{2}\right)$ and their reciprocal crosses $\left(F_{1}, F_{2}\right)$. The solid line is for males and the dotted line is for females. Genotypes corresponding to each peak are shown in the figure. $\mathrm{Jm} A 1$ and $\mathrm{Jm}$ $A 2$ are autosomal longevity genes; $\operatorname{Jm} X 1$ and $\operatorname{Jm} X 2$ are $\mathrm{X}$-chromosomal genes; $\mathrm{Jm} Y 1$ and $\mathrm{Jm} Y 2$ are genes assumed on the $\mathrm{Y}$ chromosome; $\mathrm{Jm} E 1$ and $\mathrm{Jm} E 2$ are extranuclear genes, influencing life span. parental strain, $F_{1-2} F_{2-2}, B_{1-2}, B_{1-3}, B_{1-4}, B_{2-2}$, showed a wide range of life spans, including long life spans not observed in the parental stocks.

Consequently, the results indicate that $P_{2}$ possesses a cytoplasmic factor which has a life-shortening effect which can over-ride the nuclear longevity genes, and which follows maternal inheritance accurately. This is the reason why the variance in the $F_{1}\left(V_{F_{1}}=V_{F_{1-1}}+V_{F_{1-2}}\right)$ increased, and why the above quantitative genetic analysis is invalid.

In generations where $P_{2}$ females made no contribution to the cytoplasm of the zygote, longevity graphs appeared nearly identical with those in the previous reports and their genotypic values could be likewise assigned (Yonemura et al., 1989, 1990). The nuclear genes which influenced life span to lengths far greater than those of the parental stocks, are concluded as being from the $P_{2}$ parents, in which their effects were countered by the life-shortening cytoplasmic factor. In order to account for the life spans of the flies observed here, we have to add the cytoplasmic factor, or extranuclear gene, to the $\mathrm{Jm}$ longevity gene systems observed and studied in our previous reports (Yonemura et al., 1989, 1990).

The cytoplasmic factor, or extranuclear gene, is denoted as $E 1$ for $P_{1}$ strain and $E 2$ for $P_{2}$. In the previous reports (Yonemura et al., 1989, 1990), the nuclear longevity genes for $P_{1}$ were assigned as $\mathrm{Jm} A 1$ (autosomal) and $\mathrm{Jm} X 1$ and $\mathrm{Jm} Y 1$ (sex chromosomal). In the present study, nuclear genes for $P_{2}$ were denoted $\mathrm{Jm} A 2, \mathrm{Jm} X 2$ and $\mathrm{Jm} Y 2$ (although identity of these genes with those of the $P_{2}$ strain in the previous reports is not certain). From the present experimental results, extranuclear genes are to be added to the genotypes as below:

$P_{1}$ males: $A 1 A 1 X 1 Y 1 \cdot E 1$,

$P_{1}$ females: $A 1 A 1 X 1 X 1 \cdot E 1$,

$P_{2}$ males: $A 2 A 2 X 2 Y 2 \cdot E 2$,

$P_{2}$ females: $A 2 A 2 X 2 X 2 \cdot E 2$.

Genotypes of other generations may be simply assigned. In Figs 1 and 2, the genotypes for corresponding peaks are indicated (except for $F_{2}$, to avoid congestion in the figure).

All generations possessing E2 were short-lived, irrespective of their other Jm genotypes. The shortest mode of the mortality peak in males was 27 days in the $P_{2}$, and the longest was 32 days in the $F_{1-1}$; in females, the peak commonly appeared a little later. In the generations with $E 1$, the observed segregation of life span was according to the other Jm genotypes; genotypic values could be estimated from the data in Figs 1 and 2. Taking the mode of the mortality peak as the 


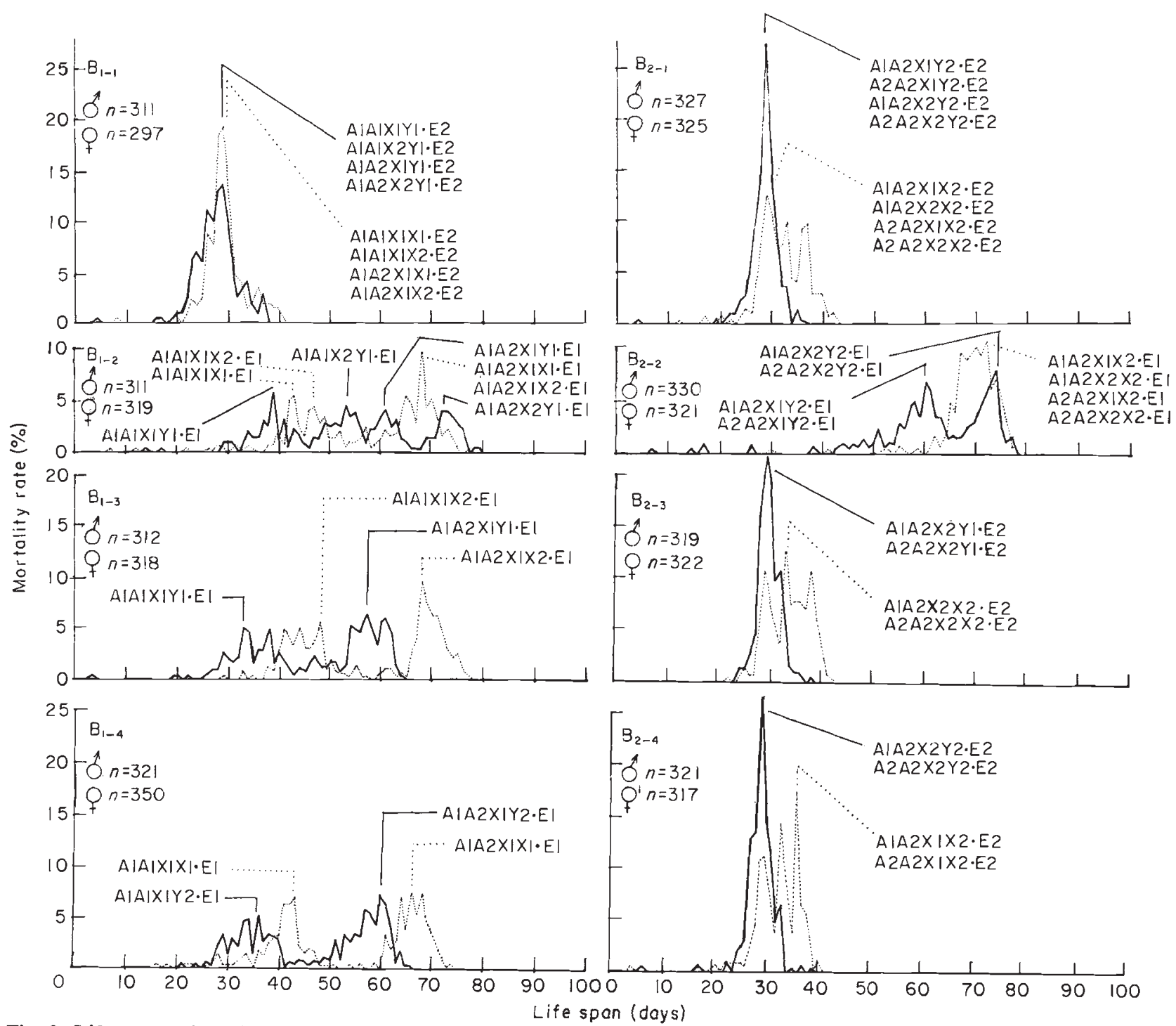

Fig. 2 Life spans of stocks of Drosophila melanogaster, where day of emergence is given as day 0. Distributions of mortality rates in backcross generations. The solid line is for males and the dotted line is for females. Genotypes corresponding to each peak are shown. $\mathrm{Jm} A 1$ and $\mathrm{Jm} A 2$ are autosomal longevity genes; $\mathrm{Jm} X 1$ and $\mathrm{Jm} X 2$ are $X$-chromosomal genes; $\mathrm{Jm} Y 1$ and $\mathrm{Jm}$ $Y 2$ are genes assumed on the $Y$ chromosome; $\mathrm{Jm} E 1$ and $\mathrm{Jm} E 2$ are extranuclear genes, influencing life span.

genotypic value, genotypes possessing $E 1$ showed the following values.

$$
\begin{aligned}
& A 1 A I X 1 Y 1=A 1 A 1 X 1 Y 2=37 \text { days }\left(P_{1}: 35, B_{1-2}: 39\right. \text {, } \\
& \left.B_{1-3}: 36, B_{1-4}: 36\right) \text {. } \\
& A 1 A 1 X 1 X 1=42 \text { days }\left(P_{1}: 39, B_{1-3}: 43, B_{1-4}: 43\right) \text {. } \\
& A I A I X 1 X 2=A 1 A 1 X 2 Y 1=50 \text { days }\left(B_{1-2}: 48\right. \text {, } \\
& \left.B_{1-3}: 48, B_{1-2}: 53\right) \text {. } \\
& A 1 A 2 X 1 Y 1=A 1 A 2 X 1 Y 2=A 2 A 2 X 1 Y 2=59 \text { days } \\
& \left(F_{1-2}: 57, B_{1-2}: 61, B_{1-3}: 57, B_{1-4}: 60\right. \text {, } \\
& \left.B_{2-2}: 58,60\right) \text {. }
\end{aligned}
$$

$A 1 A 2 X 1 X 1=66$ days $\left(B_{1-1}: 65, B_{1-4}: 66\right)$.

$A 1 A 2 X 2 Y 1=A 1 A 2 X 2 Y 2=A 1 A 2 X 1 X 2=A 1 A 2 X 2 X 2$

$=A 2 A 2 X 2 Y 2=A 2 A 2 X 1 X 2=A 2 A 2 X 2 X 2=72$ days

$\left(F_{1-2}: 69, B_{1-2}: 72, B_{2-2}: 74, B_{1-3}: 68\right)$.

From these equations, genotypic values (in days) were calculated as follows:

$Y 1=Y 2=0$,

$A 1 A 1=32$,

$A 1 A 2=52$,

$A 2 A 2=52$, 
$X 1=5$ (to $A 1 A 1)$ and 7 (to $A 1 A 2$ and $A 2 A 2$ ),

$X 2=18($ to $A 1 A 1)$ and 20 (to $A 1 A 2$ and $A 2 A 2$ ).

$Y 1$ and $Y 2$ show no effect of longevity genes. $A 2$ is dominant to $A 1$. AlA1 presents 32 days of basic life span, while $A 1 A 2$ and $A 2 A 2$ give 52 days. $X 1$ adds 5 days of supplemental life to $A 1 A 1$ and 7 days to $A 1 A 2$ and $A 2 A 2$; this effect shows a dosage effect since homozygous females live longer than hemizygous males. $X 2$ has a higher additive effect than $X 1$, adding 18 days to $A 1 A 1$ and 20 days to $A 1 A 2$ and $A 2 A 2$. In long-lived generations where segregation of life span is observed, the short-lived trait originates from $P_{1}$ and the long-lived trait from $P_{2}$. These data clearly demonstrate that the short-lived $P_{2}$ possesses nuclear longevity genes for a long life span, but an extranuclear factor suppresses their expression. Table 3 shows all the genotypic values under the control of extranuclear factor $E 1$, calculated from the above data.

The life span mode of $P_{1}$ males, the flies with the shortest life span in the presence of $E 1$ is 35 days, and the longest mode under the control of $E 2$ is 32 days. This indicates that the life-shortening effect of $E 2$ influences not only long-lived genotypes but also shortlived genotypes. That is to say, all $\mathrm{Jm}$ nuclear longevity genes are influenced by $E 2$.

The present study has demonstrated a typical maternal inheritance of short life span, indicating the presence of cytoplasmic factor which influences the expression of $\mathrm{Jm}$ longevity genes. It is inappropriate to assume that this factor is a product of the mother, such as a hormone or other metabolic substance, transmitted through cytoplasm of the ovum. If this was the case, it would be expected to produce an effect only in the $F_{1-1}$ generation $\left(P_{2}\right.$ female $\times P_{1}$ male $)$, but it could hardly leave an effect on all individuals in the $F_{2-1}$, the next generation from the $F_{1-1}$. Consequently, this factor can be assessed as a substance reproduced by the $F_{1-1}$ and transmitted through the $F_{1-1}$ females to the $F_{2-1}$, that is to say, a self-reproducing extranuclear gene, most probably part of the mitochondrial DNA (chondriome). We tentatively name the factor in $P_{1}$ as $\mathrm{Jm} E 1$ and in $P_{2}$ as $\mathrm{Jm} E 2$. Mitochondrial DNA in

Table 3 Life span given by each nuclear longevity genotype (genotypic value in days) under the influence of $\operatorname{Jm} E 1$, an extranuclear gene

\begin{tabular}{lllll}
\hline & & $\mathrm{X} 1 \mathrm{Y} 1$ & & $\mathrm{X} 1 \mathrm{X} 2$ \\
& - & $\mathrm{X} 1 \mathrm{Y} 2$ & $\mathrm{X} 1 \mathrm{X} 1$ & $\mathrm{X} 2 \mathrm{X} 2$ \\
\hline$A 1 A 1$ & 32 & 37 & 42 & 50 \\
$A 1 A 2$ & 52 & 59 & 66 & 72 \\
$A 2 A 2$ & 52 & 59 & 66 & 72 \\
\hline
\end{tabular}

D.m. has been noted to diminish with aging; Massie \& Williams (1987) noticed an association between this quantitative variation and senility.

The present experiment has shown that the $E 2$ gene has a strong suppressive effect on nuclear genes, since $A 2 A 2 X 2 Y 2$ individuals, which have a life span of 73 days with $E 1$, the longest life span of $D . m$. at $27^{\circ} \mathrm{C}$, live only 30 days with $E 2$, the shortest life span of D.m. This difference of life span is attributed to a qualitative difference between $E 1$ and $E 2$ in influencing life span, rather than a quantitative difference of mitochondrial DNA, as suggested by Massie \& Williams (1987). It is possible that a large difference of life span among species is based on differences between extranuclear longevity genes rather than between nuclear genes.

In D.m., developmental speed in the preimaginal stage has been found to have inter-relations with quantitative traits such as adult life span (Wattiaux, 1962; Burcombe \& Hollingsworth, 1970; Lints \& Lints, 1971). We have found that nuclear longevity genes control developmental speed in the preimaginal stage, from larval stage to emerging (Yonemura et al., 1991). It will be important to determine whether the extranuclear genes disclosed by the present report also influence developmental speed. It seems easier to investigate the molecular mechanisms whereby longevity genes influence developmental speed rather than to demonstrate how they determine life span. Further, now that an extranuclear, and presumably mitochondrial, factor has been shown to have a large influence on life span, it appears feasible to undertake physical manipulations, including studies involving recombinant DNA technology, with a view to altering life spans of stocks on D.m.

\section{Acknowledgment}

This study was partly supported by the Smoking Research Foundation (Japan).

\section{References}

BURCOMBE, J. V. AND HOLLINGSWORTH, M. J. 1970. The relationship between developmental temperature and longevity in Drosophila. Gerontologia, 16, 172-181.

GOODRICK, C. L. 1975. Life-span and the inheritance of longevity of inbred mice. J. Gerontol., 30, 257-263.

JOHNSON, T. E. AND wood, w. B. 1982. Genetic analysis of lifespan in Caenorhabditis elegans. Proc. Natl. Acad. Sci. USA, 79, 6603-6607.

LINTS, F. A. AND LINTS, C. V. 1971. Relationship between growth and ageing in Drosophila melanogaster. Nature (New Biol.), 229, 86-88.

LUCKINBILL, L. S., GRAVES, J. L., REED, A. H. AND KOESTAWANG, S. 1988. Localizing genes that defer senescence in Droso- 
phila melanogaster. Heredity, 60, 36\%-3\%4.

MASSIE, H. R. AND WILLIAMS, T. R. 1987. Mitochondrial DNA and life span changes in normal and dewinged Drosophila at different temperatures. Exp. Gerontol., 22, 139-153. MATHER, K. 1949. Biometrical Genetics, Methuen, London. MAYNARD SMITH, J. 1959. Sex-linked inheritance of longevity in Drosophila subobscura. J. Genet., 56, 227-235.

STORER, J. B. 1966. Longevity and gross pathology at death in 22 inbred mouse strains. J. Gerontol., 21, 404-409.

TANTAWY, A. O. AND RAKHA, F. A. 1964. Studies on natural populations of Drosophila, IV. Genetic variances of and correlations between four characters in $D$. melanogaster and D. simulans. Genetics, 50, 1349-1355.

WATTIAUX, J. M. 1962. Variation of bristle number in relation to speed of development in Drosophila melanogaster. Nature, 194, 706-707.

YONEMURA, I., ABE, M., ISHIDATE, R., ISHIYAMA, T., MOTOYAMA, T., HASEKURA, H. AND BOETTCHER, B 1990. Influence of temperature on the inheritance of adult life span in Drosophila melanogaster. Hereditas, 112, 117-127.

YONEMURA, I., MOTOYAMA, T. AND HASEKURA, H. 1989. Mode of inheritance of major genes controlling life span differences between two inbred strains of Drosophila melanogaster. Hereditas, 111, 207-214.

YONEMURA, I., MOTOYAMA, T., HASEKURA, H. AND BOETTCHER, B. 1991. Relationship between genotypes of longevity genes and developmental speed in Drosophila melanogaster. Heredity, 66, 143-149. 